\title{
Comprehensive periodic health examination: impact on health care utilisation and costs in a working population in Japan
}

\author{
Aiguo Ren, Toshiteru Okubo, Ken Takahashi
}

\begin{abstract}
Study objective - To examine the impact of a comprehensive periodic health examination programme on health care utilisation and costs in a working population. Design - A cohort method was used to observe the trends in health care utilisation and costs for a group of local government employees from 1 February 1986 until 31 January 1992, during which time (in 1988) a comprehensive periodic health examination programme had been introduced. Health care utilisation was measured by a claim rate per employee (number of claims in a year divided by number of employees) and a utilisation rate per 100 employees (number of employees who submitted at least one claim in a year divided by number of employees $\times 100)$. Health care costs were measured by claim costs per employee (total cost of claims divided by number of employees). National and prefectural changes in per capita health care costs were used for the adjustment of claim costs. Internal comparisons were made in relation to the intensiveness of the periodic health examinations administered before the introduction of the comprehensive programme.
\end{abstract}

Setting - Fukuoka Prefecture, Japan. Participants - A total of 19146 local government employees (12 455 men, 6691 women) who worked in small cities, towns, and villages in Fukuoka Prefecture throughout the study period participated. Main results - Both the claim and utilisation rates showed an increase in 1988 when the comprehensive programme was introduced. After adjustment for the national changes in per capita health care costs and the aging effect of the study participants, substantial increases in claim costs were noted after introduction of the comprehensive programme. The programme had a greater but short term effect of increasing the health care utilisation of those who had received less intensive periodic health examinations before 1988 , but no similar effect on claim costs was noted.

Conclusions - The comprehensive programme had some role in increasing health care utilisation and costs. Its effect on costs was confirmed when the general population or another population was used as a reference. When comparisons were made internally, the comprehensive programme was seen to have had a greater but short term effect of increasing the utilisation of those who had previously received less intensive health examinations to equalise the health care utilisation and costs within the study population.

( $\mathcal{F}$ Epidemiol Community Health 1994;48:476-481)

The impact of periodic health examination programmes on health care utilisation and costs is largely unknown, especially in the working population. Although there is a belief that periodic health examination can increase utilisation or costs, or both, ${ }^{2}$ little published evidence is available. In a nine year controlled trial conducted in the general population, slightly higher consultation and hospital admission rates were found in the study group which underwent two multiphasic screenings. ${ }^{3}$ In another controlled trial, also conducted in the general population, Dales $e t a l^{4}$ found no difference in the use of outpatient clinic services and hospital admissions between a study group, which was urged to take multiphasic health check ups, and a control group, which was not. This lack of difference, however, may partly stem from the fact that many of the control subjects also had check ups. ${ }^{1}$ At the end of the 11 year study period, $83.6 \%$ of the study subjects had had check ups, compared with $59 \cdot 4 \%$ of the control subjects: the average number of check ups was $5 \cdot 2$ per study subject but $2 \cdot 0$ per control subject. Barnacki et $a \bar{l}$ recently reported that the participants in a company's periodic physical examination programme used more health care resources and incurred higher costs than those who did not participate. No conclusions can be drawn from this study as to whether or not these differences were caused by the periodic physical examination, however, because of the limitation of self selection in the study design. ${ }^{6}$

In the present study, we used a cohort method to observe the trends in health care utilisation and costs over a consecutive six year period in a Japanese working population. A comprehensive periodic health examination programme was introduced during the study period and the study aimed to analyse the impact of this programme on health care utilisation and costs. 


\section{Methods}

STUDY PARTICIPANTS

The target population included all local government employees (about 22 000) working in public service sectors in small cities (that is, cities other than the four major cities of the prefecture: Fukuoka, Kitakyushu, Omuta, and Kurume, which organise their own health insurance systems), towns, and villages in $\mathrm{Fu}$ kuoka Prefecture, Japan. To ensure that the period of observation was equal, only subjects who were employed for the full study period from 1 February 1986 to 31 January 1992 were included - altogether 19146 employees.

\section{COMPREHENSIVE PERIODIC HEALTH}

EXAMINATION PROGRAMME

Periodic health examinations had been carried out in this population before 1988 but the check up items differed among working groups. Some groups received only the check up items prescribed by law. ${ }^{7}$ These items included the following: a medical and work history, physical examination, height (excluded for those 25 years of age or older) and weight measurement, visual acuity and audibility testing, chest $x$ ray, sputum examination (excluded for those with no abnormalities seen on chest $x$ ray), blood pressure measurement, and urine analysis (glucose and protein; excluded for those under 40 years of age). Some groups received various additional items, such as blood lipid determination (total cholesterol and triglycerides), hepatic function test (glutamic-oxaloacetic transaminase, glutamic-pyruvic transaminase, $\gamma$-glutamyl transpeptidase), $x$ ray examination of the upper gastrointestinal tract, electrocardiogram, abdominal ultrasonography, or funduscopy. Two groups (with a total of 55 employees) had no periodic health examination at all.

In 1988, the periodic health examinations were reorganised into a new programme that was the same for all working groups - the comprehensive periodic health examination.
This was conducted annually as a group health service for all the employees. Table 1 lists the check up items in relation to age group. The number of items included in the new programme was higher than that in the previous examinations. Many of the items aim to early detect cancers (for example, gastric cancer and lung cancer, which account for the most cancer deaths) and some chronic diseases (for example, cerebro-cardiovascular diseases, which account for most non-cancer deaths).

During the six year period, the participation rates for the comprehensive programme ranged from $96 \cdot 3-98 \cdot 4 \%$. Various abnormalities were found in $57.0-70.3 \%$ of the participants. Of these, $40 \cdot 8-44 \cdot 4 \%$ were recommended to have more sophisticated check ups, and $31 \cdot 0-45 \cdot 7 \%$ of those recommended further investigation still had abnormal results.

The study participants' health care utilisation and costs, other than those included in the comprehensive programme, were identified through a health insurance organisation - the Fukuoka Prefecture Local Public Service Mutual Aid Association.

\section{LOCAL PUBLIC SERVICE MUTUAL AID} ASSOCIATION

This insurance plan was established specifically for employees who work in public service sectors of the local government. By Japanese law, all of these employees are covered exclusively by this plan. Each of the 47 prefectures of Japan has one organisation to cover the local government employees in small cities, towns, and villages within the prefecture.

This is a self insurance plan, with half of the financial resources being contributed by the insured employees (a premium of about $4 \%$ of their salaries) and half by the employers. The national government pays the administrative expense. Employees pay $10 \%$ of the total costs for health care utilisation; the rest is covered by the insurance. If an employee's share of the costs for an illness exceeds a certain amount

Table 1 Items checked in the comprehensive periodic health examination in relation to age group

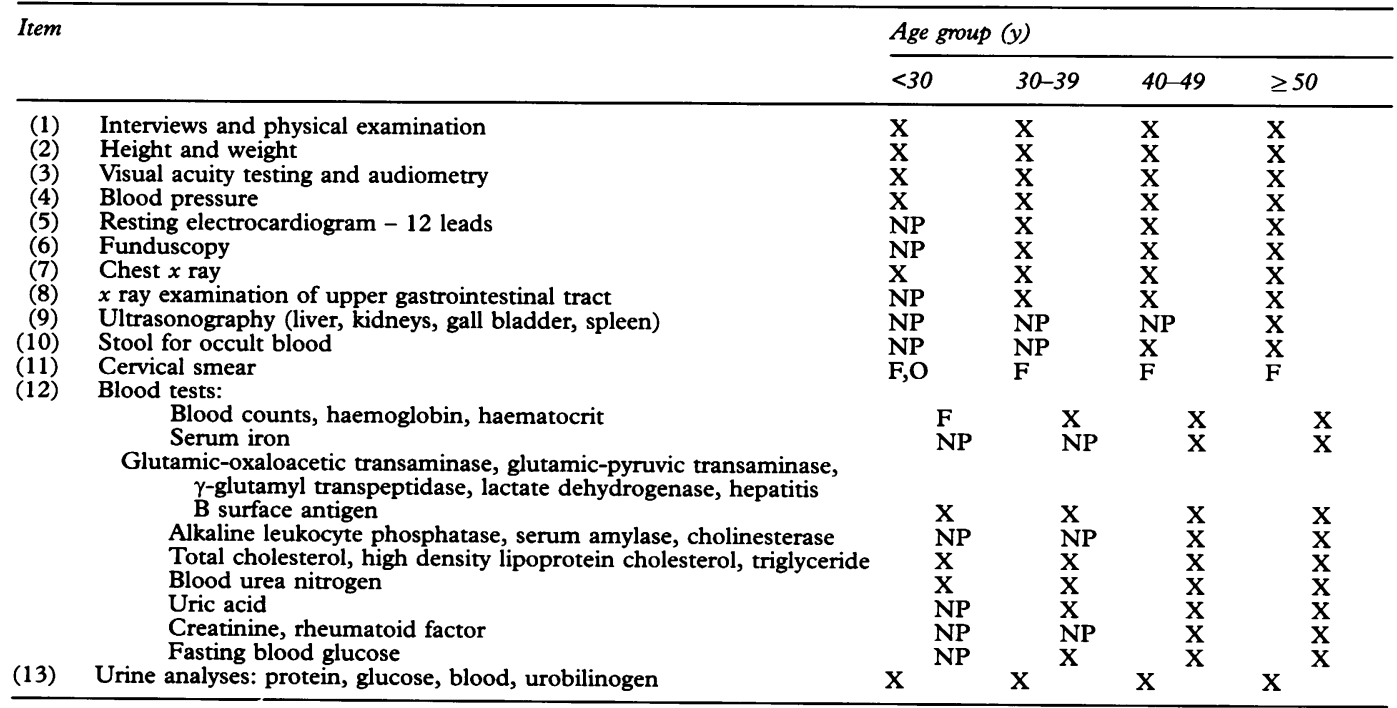

$\mathrm{X}=$ prescribed for both genders; $\mathrm{NP}=$ not provided; $\mathrm{F}=$ female only; $\mathrm{O}=$ optional. 
in a month (54000 yen before June 1989, 57000 yen from June of 1989 to April 1991, and 60000 yen thereafter) he or she is reimbursed the excess. ${ }^{8}$ No restrictions are imposed on employees with regard to the type of medical institution, disease category, frequency of utilisation, or costs. Therefore nearly all of the health care utilisation and costs can be identified through this insurance.

The analysis was based on the insurance claims submitted by the study participants. The claim data were provided by the above mentioned organisation on magnetic tapes. To protect privacy, employees names were deleted from the claim data files and a seven digit number was used to identify the claim(s) submitted by each employee.

\section{ANALYTICAL METHODS}

The number of claims submitted by each employee was counted and the amount of money was summed on a year to year basis. Health care utilisation was measured by two indices a claim rate per employee (the number of claims in a year divided by number of employees) and a utilisation rate per 100 employees (the number of employees who submitted at least one claim in a year divided by number of employees times 100). Health care costs were measured by the claim costs per employee - the total cost of claims in a year divided by number of employees. These costs included the $10 \%$ borne by the employees themselves. If an employee did not submit any claim, the number of claims and the amount of money charged were assigned a value of zero.

The observed indices in 1988 and thereafter were compared with the corresponding indices predicted, based on data from 1986 to 1987 . The $t$-test was used to test the differences in claim rate and in claim costs, and the $\chi^{2}$ test was used for the utilisation rate.

Two major external factors may influence the health care costs - inflation of health care costs and the aging of the study subjects. National $^{8}$ and prefectural changes in per capita health care costs from 1986-91 were used to adjust for the influence of inflation. Specifically, the proportion of health care costs attributed to the national or prefectural changes in per capita health care costs was deducted from the claim costs per employee each year. As the mean age of the study participants grew faster than that of the general population (one year for the study participants per year of observation, but about 0.25 year for men and 0.30 year for women in the general population each year during the period $1980-91^{9}$ ), additional adjustment was made after the claim costs had been adjusted for the national changes. No similar adjustment was made for claim rate and utilisation rate because of lack of relevant national data.

To examine any possible difference in the effects of the comprehensive health programme on those who received less intensive or no health examinations and on those who received relatively intensive health examinations before 1988 , we classified the study subjects into two groups by using per capita expense on the examinations as a proxy of the intensiveness. Those with expenses of less than 4000 yen, the average expense of the items required by law, were referred to as the less intensive group ( $n=$ 8755 ), and those with expenses of 4000 yen or over were referred to as the intensive group ( $\mathrm{n}=$ 9291). About 1100 (6\%) employees' expenses remained unknown and these employees were excluded from this part of the analysis. The above three indices were compared between the two groups by means of analysis of variance controlling for baseline age, sex, and occupation differences. Because the data were for the same group of subjects over the entire six year period, the repeated measures analysis was used. ${ }^{1011}$

\section{Results}

At the beginning of observation ( 1 February $1986)$, the mean (SD) age was $37 \cdot 3(8 \cdot 4)$ years for the 12455 men and $37 \cdot 5(8 \cdot 0)$ years for the 6691 women. The distribution of the study participants in relation to age and sex is shown in table 2.

Figure 1 shows the trends in the claim rate per employee. A constant increase was seen throughout the study period, with a peak in 1988, the year the comprehensive programme was introduced. The observed claim rates were significantly higher than the predicted rates in $1988,1990$, and 1991 ( $p<0 \cdot 001)$, but not in $1989(p=0.3746)$. The utilisation rate also showed an excess increase in 1988 (fig 2). This increase was relatively sharp with an additional increase of about 1.2 (persons who utilised health care per 100 employees) over the predicted utilisation $(p<0.0001)$. Thereafter the

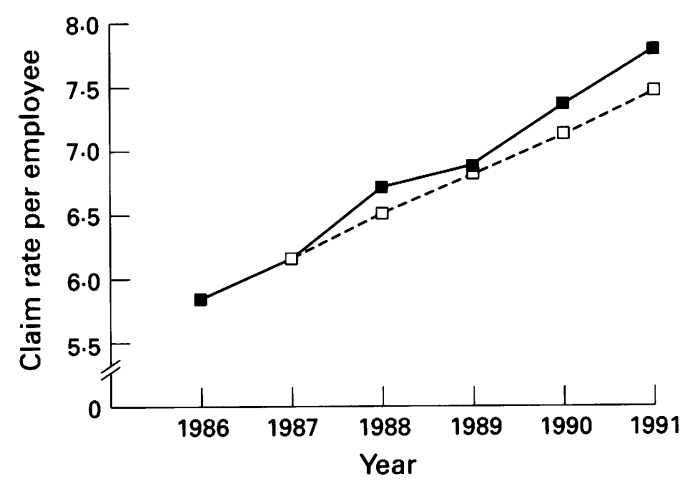

Figure 1 Claim rate per employee (number of claims in a year/number of employees): - - observed claims; - $\square--$ predicted claims based on data from 1986-87.

Table 2 Distribution of the study participants in relation to age (on February 1, 1986) and sex

\begin{tabular}{|c|c|c|c|c|}
\hline \multirow[t]{2}{*}{ Age (y) } & \multicolumn{2}{|l|}{ Men } & \multicolumn{2}{|c|}{ Women } \\
\hline & No & $(\%)$ & No & $(\%)$ \\
\hline $\begin{array}{l}<30 \\
30-39 \\
40-49 \\
\geq 50\end{array}$ & $\begin{array}{l}2518 \\
5771 \\
2937 \\
1229\end{array}$ & $\begin{array}{c}(20 \cdot 2) \\
(46 \cdot 3) \\
(23 \cdot 6) \\
(9 \cdot 9)\end{array}$ & $\begin{array}{r}1247 \\
2985 \\
1947 \\
512\end{array}$ & $\begin{array}{r}(18.6) \\
(44.6) \\
(29 \cdot 1) \\
(7 \cdot 7)\end{array}$ \\
\hline Total & \multicolumn{2}{|c|}{$12455(100 \cdot 0)$} & \multicolumn{2}{|c|}{$6691(100 \cdot 0)$} \\
\hline
\end{tabular}




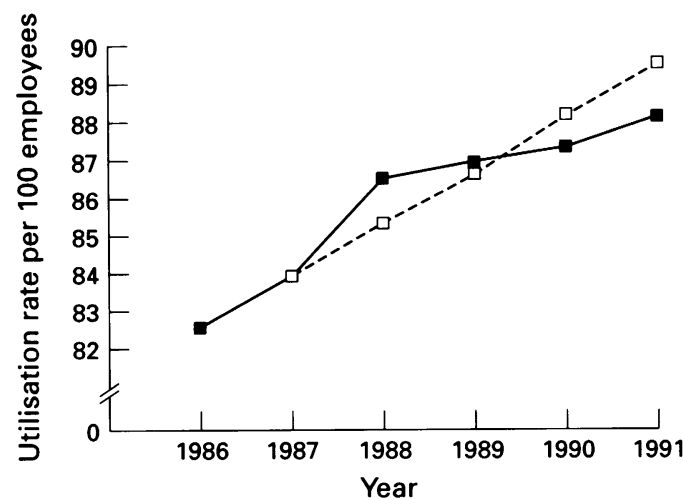

Figure 2 Utilisation rate per 100 employees I(number of employees who submitted at least one claim in a year/ number of employees) $\times 100 \mathrm{~J}:-\mathbf{\square}-$ observed claims; $--\square--$ predicted claims based on data from 1986-87.

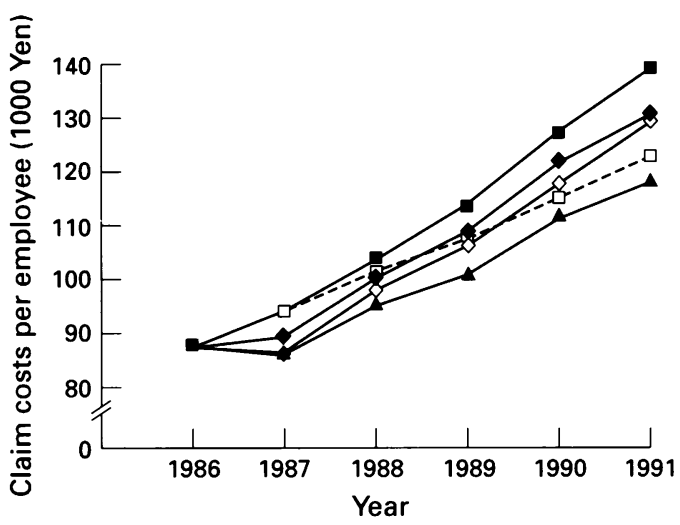

Figure 3 Claim costs per employee: - $\square-$ observed costs; - - $\square--$ predicted costs based on data from costs; - $\square--$ predicted costs based on data from $-\diamond-$ costs adjusted for prefectural changes; $-\mathbf{\Delta}-$ costs adjusted for aging.

increase became moderate, but the utilisation rate stayed high.

Figure 3 shows the trends in claim costs per employee. In the unadjusted figures, larger increases were seen in 1988 and thereafter, compared with 1987. The excess increases in the observed over the predicted claim costs were significant in 1989 and thereafter $(p<0.01)$, with an insignificant excess of 2931 yen $(p=0.1207)$ in 1988 .

When the claim costs were adjusted for national changes, a different pattern emerged. The adjusted line showed only a slight increase from 1986 to 1987 , which can be regarded as the effect of one year of aging of the study participants. In contrast, from 1988 until 1991, there were large increases, which reflect rhe effect of subjects' aging and, most importantly, the probable effect of the comprehensive periodic health examination. In the adjustment for aging, we assumed that the increase in health care costs attributable to aging was:

\section{$\mathrm{N} \times$ increases from one year aging}

where $\mathbf{N}$ denotes the increased age $(\mathrm{s})$ from the baseline year of 1986. Increases from one year of aging were estimated from the slope between 1986 and 1987 in the line that had been adjusted for the national changes. This means that the proportion of claim costs attributable

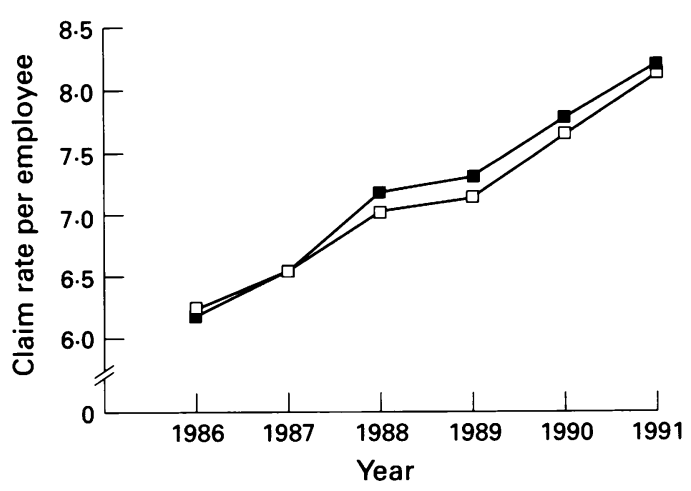

Figure 4 Claim rate per employee in relation to the intensiveness of the periodic health examination administered before 1988: ——- less intensive group; $-\square--$ intensive group.

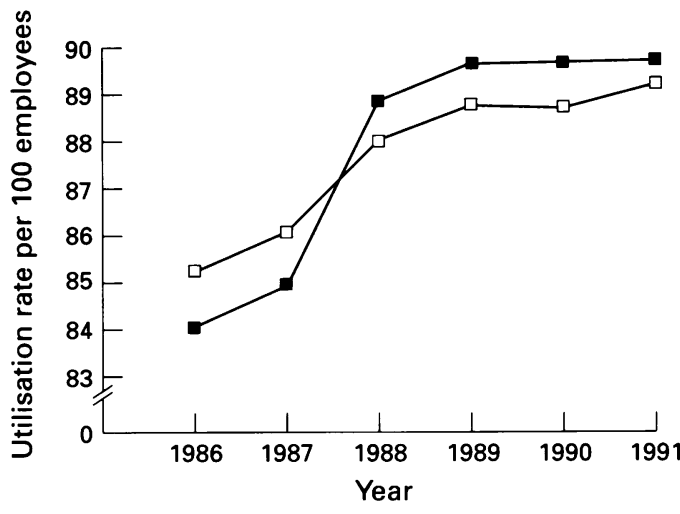

Figure 5 Utilisation rate per 100 employees in relation to the intensiveness of the periodic health examination administered before 1988: — - - less intensive group; $-\square-$ intensive group.

to aging was 0.0295 in $1987,2 \times 0.0295$ in $1988, \ldots$, and $5 \times 0.0295$ in 1991 . After these were used for further adjustment of claim costs that had already been adjusted for the national changes, the adjusted line still showed substantial increases compared with the adjusted claim costs in 1987. The net increases were 9061 yen in $1988(\mathrm{p}<0.05), 14798$ yen in 1989 $(\mathrm{p}<0.01), 25603$ yen in $1990(\mathrm{p}<0.0001)$, and 32475 yen in 1991 ( $p<0.0001)$, which can be attributed to the comprehensive programme.

As an alternative, the claim costs were adjusted by using the annual changes in per capita health care costs from another health insurance plan in the same prefecture. The trend line adjusted for the prefectural changes was similar to the line adjusted for the national changes, except that in the former, the adjusted claim costs in 1987 were lower than the claim costs in 1986 (86360 $v 87092$ yen).

Different effects of the comprehensive periodic health examination on the claim rate were noted between the less intensive group and the intensive group (fig 4). Although the claim rates were not significantly different between the two groups when averaged over the six year period ( $p=0 \cdot 3826)$, a larger increase in the less intensive group was detected in 1988 ( $p=$ 0.0265 ). The less intensive group also showed a larger increase in the utilisation rate in 1988 $(p=0.0029)$ (fig 5). This increase reversed the 


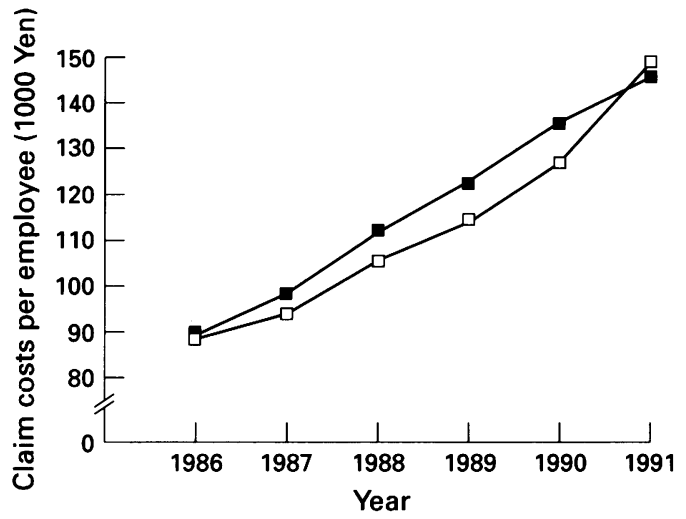

Figure 6 Claim costs per employee in relation to the intensiveness of the periodic health examination administered before 1988: - $\square-$ less intensive group $-\square-$ intensive group.

order in utilisation rate between the two groups. However, both the claim rate and the utilisation rate were coming closer to each other in 1991 - that is after four years of the programme.

No significant difference in the claim costs were found between the less intensive and the intensive groups, either averaged over the six year period $(p=0 \cdot 1552)$ or compared between 1988 and 1987 ( $p=0.6362$ ) (fig 6). The claim costs of the two groups were virtually the same in 1991.

\section{Discussion}

In the analysis of the claim and utilisation rates, the observed values were compared with the predicted values. The predicted values were projected from the 1986-87 data, assuming that the two rates would rise in the same manner as they had done in the previous year before the introduction of the comprehensive health programme. The utilisation rate, however, is unlikely to rise constantly in a linear way because it has a top limit, $100 \%$. If we assume that the utilisation rate rises linearly in the short term, however, we can accept that there were excess increases in the utilisation rate, at least in 1988. Excess increases in the claim rate were noted in all years except 1989 after the introduction of the comprehensive health programme.

The comprehensive programme had a clear effect on the utilisation rate in 1988. This means that some employees, who otherwise would not have used health care, were found to have abnormalities at check up and were therefore recommended to have further check ups or follow up treatment. The effect on the claim rate, on the other hand, seems very weak. However, this may not mean that the comprehensive health programme had little effect in increasing the frequency of utilisation. This is because of the fact that the claims are released on a monthly basis, not on a frequency basis. If an employee uses a clinic or the same department of a hospital several times in a specific month, only one claim is generated. That is, additional utilisation may not incur an additional number of claims. The actual effect of the comprehensive programme on the frequency of utilisation would be much greater than that observed from the claim rate if the claims had been released on a frequency basis.

Using the national or prefectural changes in per capita health care costs to adjust the claim costs enabled us to adjust for inflation of health care costs, aging of the general population, and other unknown factors that may influence the health care costs. Statistics show that regional variations in per capita health care costs are large, and Fukuoka Prefecture is located in an area where per capita health care costs are high. ${ }^{8}$ Thus prefectural data may be preferable. The prefectural data used in this study are from another insurance plan which covers a much larger population (over 1.2 million). No periodic health examination is provided for this population. This insurance plan includes some retired employees, and the average age is higher than that of our study population. This means that using this insurance plan as a reference includes the possibility of over-adjustment of the claim costs. The fact that the adjusted claim costs in 1987 were lower than in 1986 confirmed this. Nevertheless, the adjusted costs still showed substantial increases beginning in 1988 when the comprehensive programme was introduced.

Because the study population was aging at a much faster rate than the general population, we put extra weight on aging in the adjustment. This leaves the comprehensive health programme as the only factor responsible for the increases in the trend line adjusted for both national changes in health care costs and aging of the study subjects.

The comprehensive health programme had a greater but short term effect on the utilisation rate of those who had received less intensive periodic health examinations before its introduction, but no similar effect on the costs of claims was noted. After four years, both the utilisation and costs within the population tended to be equal.

The proportions of subjects with abnormal findings detected at comprehensive health programme check ups seem extremely high. "Abnormal" in this programme was defined very broadly, however: the subjective judgment of the attending physician or any laboratory test result that was outside the normal range. In fact, less than half of those with abnormal findings were considered to deserve medical attention and referred for further investigation. The final proportion of those with abnormalities that required serious medical attention ranged from $7 \%(57 \% \times 40.8 \% \times$ $31.0 \%)$ to $14 \%(70.3 \% \times 44.4 \% \times 45.7 \%)$ of all the participants. In view of the large number of check up items and the aim of the programme - that is, "total health" of the employees, as opposed to checking for a single specific disease as in many other screening programmes, the proportion does not seem so high. However, being labelled with something "abnormal" in a given test, some of the employees may have sought further consultation for reassurance, even if their condition was minor and did not require such. 
Two other features of the comprehensive programme - the yearly checks and the high participation rate - may also be related to its effects on health care utilisation and costs and account for the difference with other studies ${ }^{34}$ in which both the frequency and the participation rate were low.

The findings of this study are reinforced by the results of some health promotion programmes $^{1213}$ in which health or risk screenings were found to have some effect of increasing health care utilisation and costs.

This analysis has three possible limitations. Firstly, no adequate control groups could be allocated. If some other events affected solely the study population during the study period, the conclusion might be subject to bias. Interviews with the concerned personnel did not, however, reveal any such events. Secondly, the factors used in the further adjustment for aging were somewhat arbitrary. The true effect of aging may be greater than that used. However, it is difficult to attribute all the increases in the adjusted trend to the aging effect. Finally, the study included only those who were working for the full study period and it is not clear if there were systematic differences in health status, and therefore in health care utilisation and costs, between the study participants and those not included. The person-year method is a way of taking the latter into consideration, but it requires reliable data that record precisely the movements of the population. Unfortunately, no such data are available.

In summary, the comprehensive programme played some part in increasing health care utilisation and costs. The effect on costs remained after inflation had been controlled for. When comparisons were made within the population, the programme had a greater effect of increasing the health care utilisation of those who received less intensive periodic health examinations than that of those who received intensive health examinations before the introduction of the programme., but the pro- gramme had no effect of increasing the former's health care costs. After four rounds of implementation, the programme tended to equalise the health care utilisation and costs within the population.

This study is a preliminary analysis and further studies are needed to confirm its impact on health care utilisation and costs, preferably by using a comparable population in which no such programme is administered as a comparison group. In addition, the observation should be extended to a longer period. We believe that these findings are not necessarily criticisms of the programme; rather, they are helpful to health policy makers, health promoters, and administrators planning such programmes.

The authors thank Ms Rie Nakamura and Ms Michiko Fukumitsu for their help in preparing the data.

1 Rose SD. The periodic health examination. Prim Care 1980; 7:653-65.

2 Sawyer HJ. More on physical examination programs (letter). f Occup Med 1989;31:695.

3 The South-East London Screening Study Group. A controlled trial of multiphasic screening in middle-age: results of the South-East London Screening Study. Int $f$ Epidemiol 1977;6:357-63.

4 Dale LG, Friedman GD, Collen MF. Evaluating periodic multiphasic health checkups: A controlled trial. Fournal of Chrnic Diseases 1979;32:385-404.

5 Bernacki EJ, Tsai SP, Malone RD. Participation in a periodic physical examination program and group health care utilphysical examination program and group health care

6 Sherman MS. Periodic physical examination programs (letter). F Occup Med 1989;31:693-4.

7 Tsuchiya K. Development of occupational health in Japan. fournal of University of Occupational and Environmental Health. 1991;13:191-205.

8 Department of Statistics and Information, Ministry of Health and Welfare. National medical costs. Tokyo: Health and Welfare Statistics Association, 1992. (In Japanese).

9 Shigematsu T. The Japanese life span. Fapanese fournal of Public Health 1993;40(10):3-7. (In Japanese).

10 SAS Institute Inc. SAS/STAT user's guide. Version 6, 4th ed, Vol 2. Cary, NC: SAS Institute Inc, 1989;891-996.

11 Littel C, Freund RJ, Spector PC. SAS systems for linear models. 3rd ed. Cary NC: SAS Institute Inc, 1991;265291

12 Bly JL, Jones RC, Richardson JE. Impact of worksite health promotion on health care costs and utilization: Evaluation of Johnson \& Johnson's Live for Life Program. $\mathfrak{F} A M A$ 1986;256:3235-40.

13 Gibbs JO, Mulvaney D, Henes C, Reed RW. Work-site health promotion: Five-year trend in employee health care costs. f Occup Med 1985;27:826-30. 